\title{
Image Registration and Wavelet Based Hybrid Image Fusion
}

\author{
Mrs.Disha S Bhosle ${ }^{1,}$ Mrs. Kanchan S Gorde ${ }^{2}$ \\ ${ }^{\prime}$ (Departmen Of Electronic, Atharva College Of Engineering,India) \\ ${ }_{2}^{2}$ (Department Of Electronic, Terna College Of Engineering,India)
}

\begin{abstract}
Image fusion is to integrate complementary multisensor, multitemporal and multiview data into one new image containing data of quality which cannot be achieved otherwise. As optical lenses in capturing device have limited depth-of-focus, it is sometime not possible to obtain an image that contains all relevant objects, in focus. To achieve all objects in focus, a fusion process is required which focuses all objects.The solution to this problem is the new architecture called Hybrid Image Fusion which involves physical image alignment by using image registration, then applying DWT. In the intermediate stage the images are decomposed and further fused to obtained hybrid image by using pixel rules.
\end{abstract}

Keywords: D.W.T, Image Registration, Hybrid Image Fusion, Multiresolution, Pixel rules

\section{INTRODUCTION}

Image fusion is the combination of information gathered from multiple images of the same scene and the result of fusion is a new image which is more suitable for human perception or further image -processing tasks. Image fusion can be performed at different levels of the information representation. Due to imperfections of imaging devices (optical degradations, limited resolution of CCD sensors) and instability of the observed scene (object motion, media turbulence), acquired images are often blurred, noisy and may exhibit insufficient spatial or temporal resolution..Image fusion tries to solve this to combine information from several images (sensors) taken from the same scene in order to achieve a new fused image, which contains the best information coming from the original images. Hence, the fused image has better quality than any of the original images.

The fused image is more suitable for the purposes of object detection, target recognition and human visual perception. In general, based on different extracting extents of the information, image fusion can take place at pixel, feature, or decision level[1]. The most common approach of image fusion, known as pixel-based fusion, consists of comparing information among the pixels in the same location or pixels in the same region in different images. So far, pixel-based fusion has attracted much attention and many interrelated methods have been proposed such as weighted means and multi-resolution analysis. Multi-resolution transformations have been recognized as a very useful approach to analyses the information content of images for the purpose of image fusion. Multi-resolution fusion uses pyramid or wavelet transform at multiscale for the representation of the source images [5].

\subsection{Image Registration}

In image fusion, it is essential that the image information from all the constituent images be adequately aligned and registered prior to combining the images, ensuring that the information from each sensor is referring to the same physical structures in the environment [2]. This is a key issue in image fusion, as a misalignment produces severe edge artifacts in the combined images. This is particularly significant in images where the edges are abundant. There are several approaches that have been investigated for alignment of multiple digital images . Image registration is the process of overlaying two or more images of the same scene taken at different times, from different viewpoints, and/or by different sensors [3]. It geometrically aligns two images - the reference and sensed images. The present differences between images are introduced due to different imaging conditions. For the task of IR, a set of control points can be derived from the extraction of features in the image [1].

Registration is necessary in order to be able to compare or integrate the data obtained from these different measurements. The majority of the registration methods consist of the following four steps:-

1.1.1 Feature detection: - Salient and distinctive objects (closed-boundary regions, edges, contours, line intersections, corners, etc.) are manually or, preferably, automatically detected. For further processing, these features can be represented by their point representatives (centers of gravity, line endings, distinctive points), which are called control points (CPs) in the literature [3].

1.1.2 Feature matching: - In this step, the correspondence between the features detected in the sensed image and those detected in the reference image is established. Various feature descriptors and similarity measures along with spatial relationships among the features are used for that purpose [4]. 
1.1.3 Transform model estimation: - The type and parameters of the so-called mapping functions, aligning the sensed image with the reference image, are estimated [4]. The parameters of the mapping functions are computed by means of the established feature correspondence.

1.1.4 Image resampling and transformation: - The sensed image is transformed by means of the mapping functions. Image values in non-integer coordinates are computed by the appropriate interpolation technique [3] [4].

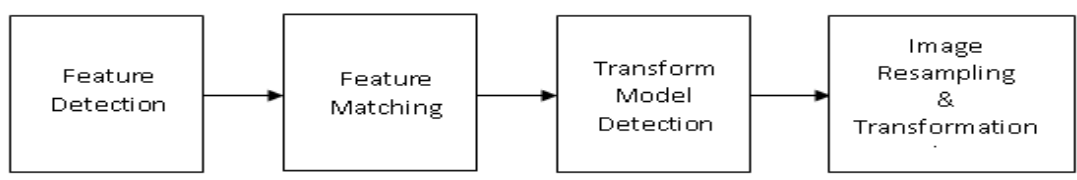

Fig 1.Concept of Image Registration

\subsection{Discrete Wavelet Theory}

Wavelets are finite duration oscillatory functions with zero average value. The irregularity and good localization properties make them better basis for analysis of signals with discontinuities. Wavelets can be described by using two functions viz. the scaling function $\mathrm{f}(\mathrm{t})$, also known as 'father wavelet' and the wavelet function or 'mother wavelet'. 'Mother' wavelet $\psi(\mathrm{t})$ undergoes translation and scaling operations to give self similar wavelet families as in (1) [5].

$$
\psi_{a b}(t)=\frac{1}{\sqrt{a}} \psi\left(\frac{t-b}{a}\right),(a, b \varepsilon R), a>0
$$

Where $\mathrm{a}$ is the scale parameter $\mathrm{B}$ the translation parameter



Fig 2. Two dimensional sub band coding algorithms

The 2-D wavelet analysis operation shown in Fig 2 consists of filtering and down-sampling horizontally using the 1-D low pass filter L (with impulse responses l(i)) and high pass filter $\mathrm{H}$ (with impulse responses $\mathrm{h}(\mathrm{j})$ ) to each row in the image I $(\mathrm{x} ; \mathrm{y})$, producing the coefficient matrices $\mathrm{IL}(\mathrm{x} ; \mathrm{y})$ and $\mathrm{IH}(\mathrm{x}$; $\mathrm{y})$.Vertically filtering and down-sampling follows, using the low pass and high pass filters $\mathrm{L}$ and $\mathrm{H}$ to each column in IL(x; y) and IH (x; y) and produces four sub images ILL(x; y),ILH (x; y), IHL(x; y) and IHH (x; y) for one level of decomposition.ILL $(x ; y)$ is a smooth sub image corresponding to the low-frequency band of the MSD and can be considered as a smoothed and sub sampled version of the original image I (x; y), i.e. it represents the coarse approximation of I $(x ; y)$. ILH $(x ; y)$ IHL $(x ; y)$ and IHH $(x ; y)$ are detail sub images, which represent the horizontal, vertical and diagonal directions of the image $\mathrm{I}(\mathrm{x} ; \mathrm{y})[1]$.

\subsection{Implemented Fusion Rules}

Let $\mathrm{I}_{1}(\mathrm{x}, \mathrm{y})$ and $\mathrm{I}_{2}(\mathrm{x}, \mathrm{y})$ are images to be fused, the decomposed low frequency sub images of $\mathrm{I}_{1}(\mathrm{x}, \mathrm{y})$ and $\mathrm{I}_{2}(\mathrm{x}, \mathrm{y})$ are $\mathrm{LI}_{1} \mathrm{p}(\mathrm{x}, \mathrm{y})$ and $\mathrm{LI}_{2} \mathrm{p}(\mathrm{x}, \mathrm{y})$. Decomposed high frequency sub-images of $\mathrm{I}_{1}(\mathrm{x}, \mathrm{y})$ and $\mathrm{I}_{2}(\mathrm{x}, \mathrm{y})$ are $\mathrm{HI}_{1} \mathrm{p}, \mathrm{k}(\mathrm{x}, \mathrm{y})$ and $\mathrm{HI}_{2} \mathrm{p}, \mathrm{k}(\mathrm{x}, \mathrm{y})$. ( $\mathrm{p}$ and $\mathrm{k}$ is the parameter of resolution, where $\mathrm{p}=1,2,3 \ldots . \mathrm{P}$ for every $\mathrm{p}$ and $\mathrm{k}=1,2,3$ $\ldots k$ for every $k[6] .:$ The below mentioned fusion rules were implemented with discrete wavelet transform for image fusion :- 


\subsubsection{Maximum selection}

The greater the pixel values the more in focus the image. Thus this algorithm chooses the in-focus regions from each input image by choosing the greatest value for each pixel, resulting in highly focused output[8].

\subsubsection{Averaging scheme}

$$
\mathrm{F}(\mathrm{p}, \mathrm{k})=\max \left(\mathrm{I}_{1} \mathrm{p}, \mathrm{k} \mathrm{I}_{2} \mathrm{p}, \mathrm{k}\right) \& \mathrm{~F} \mathrm{P}=\max \left(\mathrm{LI}_{1} \mathrm{p}, \mathrm{LI}_{2} \mathrm{p}\right)
$$

This is the simple way of obtaining an output image with all regions in focus.The values of the pixel P ( $\mathrm{i}, \mathrm{j}$ ) of each image is taken and added .This sum is then divided by 2 to obtain the average. The average value is assigned to the corresponding pixel of the output image which is given in equation (3). This is repeated for all pixel values.

\subsubsection{Averaging and absolute scheme}

$$
\mathrm{F}(\mathrm{p}, \mathrm{k})=\left(\mathrm{I}_{1} \mathrm{p}, \mathrm{k}+\mathrm{I}_{2 \mathrm{p}}, \mathrm{k}\right) / 2 \& \mathrm{~F} \mathrm{P}=\left(\mathrm{LI}_{1} \mathrm{p}+\mathrm{LI}_{2} \mathrm{p}\right) / 2
$$

It uses averaging to fuse low frequency sub-bands and selects the absolute maximum value for high frequency bands.

\subsubsection{Pixel diagonal averaging/absolute}

$$
\mathrm{F}(\mathrm{p}, \mathrm{k})=\max \left(\operatorname{abs}\left(\mathrm{I}_{1} \mathrm{p}, \mathrm{k}\right), \operatorname{abs}\left(\mathrm{I}_{2} \mathrm{p}, \mathrm{k}\right)\right) \& \mathrm{~F} \mathrm{P}=\left(\mathrm{LI}_{1} \mathrm{p}+\mathrm{LI}_{2} \mathrm{p}\right) / 2
$$

It consider the average of approximation and diagonal details of source image to form the LL and HH sub band and uses absolute maximum rule to form LH and HL sub bands of fused image.

\section{The Proposed Methodology}

This paper proposes an hybrid image fusion using the above mentioned rules, it consider all the relevant information from the two images and finally produces one single image which incorporates the best information from both images. Following is the algorithm for hybrid image fusion:-

1) Consider two set of unregistered images i.e image $I_{1}$ and $I_{2}$.

2) Align the two images by using image registration,i.e by using geometric transform

3) Once the images are align to their physical appearance then perform Diceretre Wavelet transform,so image which are futher sub divided to obtain LL,HH,HL,LH Subbands.

4) The LL subband has lot of information of edges and color.so this subband is further used for multilevel decomposition by the haar transform or coiflet transform.

5) In this step the fusion rules are applied to the subbands and finally the IDWT is done .

6) The IDWT of fused image 1 and IDWT of fused image 2 is fused to obtain final fusion of images. The image finally obtained is a image which has infocus details of both the images.

7) Evaluate the result images by using the M.S.E,P.S.N.R and S.S.I.M .

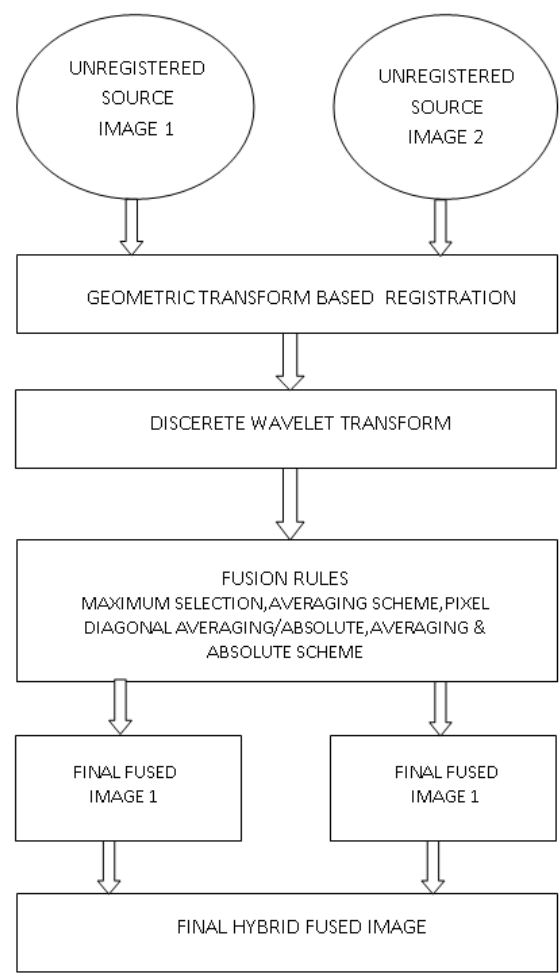

Fig No.3. The flow diagram of Hybrid Image Fusion 


\section{Result}

The results obtained are evaluated by using the Mean Square Error(M.S.E),Peak Signal To Noise Ratio (P.S.N.R) and Structural Similarity Index(SSIM).The results obtained by using the hybrid fusion rules show removal of noise and artifacts from the final fused image, it preserves the boundary information and structural details. The final hybrid images are been evaluated by following Equations (5),(6)and(7).

\subsection{Mean Square Error (M.S.E):-}

The following Mathematical equation (5) is used to evaluate Mean Square Error[5].

$$
\begin{array}{r}
\text { M.S.E }=\frac{\sum_{I=1}^{M} \sum_{j=1}^{N}(S(i, j)-F(i, J))^{2}}{M \times N} \\
\text { Where } \mathrm{S} \text { is the source image } \\
\mathrm{F} \text { is the Fused image }
\end{array}
$$

\subsection{Peak Signal To Noise Ration (PSNR):-}

PSNR is the ratio between the maximum possible power of a signal and the power of corrupting noise that affects the fidelity of its representation [5].The PSNR measure is given by:-

$$
P S N R=10 \log _{10}\left(\frac{255^{2}}{M S E}\right)
$$

\subsection{Structural Similarity Index(SSIM) :-}

The Structural Similarity (SSIM) index is a method for measuring the similarity between two images; it is a measure of image quality based on an initial uncompressed or distortion - free image as reference.The SSIM is calculated on various windows of an image[7]. The measure betwwn two windows $\mathrm{x}$ and $\mathrm{y}$ of common size $\mathrm{N} \times \mathrm{N}$ is:-

$$
\operatorname{SSIM}(x, y)=\frac{\left(2 \mu_{x} \mu_{y}+c_{1}\right)\left(2 \sigma_{x y}+c_{2}\right)}{\left(\mu_{x}^{2}+\mu_{y}^{2}+c_{1}\right)\left(\sigma_{x}^{2}+\sigma_{y}^{2}+c_{2}\right)}
$$

\begin{tabular}{|c|c|c|c|c|c|c|c|c|c|c|c|c|}
\hline \multirow{2}{*}{$\begin{array}{l}\text { Source } \\
\text { image }\end{array}$} & \multicolumn{4}{|c|}{ M.S.E } & \multicolumn{4}{|c|}{ P.S.N.R } & \multicolumn{4}{|c|}{ S.S.I.M } \\
\hline & Haar & DB4 & Coif1 & Sym2 & Haar & DB4 & Coif1 & Sym2 & Haar & DB4 & Coif1 & Sym2 \\
\hline $\begin{array}{c}\text { Two } \\
\text { Books }\end{array}$ & 14.05 & 13.16 & 13.60 & 13.09 & 8.53 & 8.22 & 8.68 & 8.84 & 0.953 & 0.941 & 0.945 & 0.95 \\
\hline $\begin{array}{c}\text { Two } \\
\text { Clocks }\end{array}$ & 9.52 & 9.12 & 9.18 & 9.22 & 10.63 & 10.82 & 10.79 & 10.77 & 0.91 & 0.913 & 0.912 & 0.915 \\
\hline
\end{tabular}

The performance of various images is shown below in Table No.1 which compares the different types of filter and values of MSE,PSNR,SSIM.

Table No.1 shows various values of MSE, PSNR,SSIM
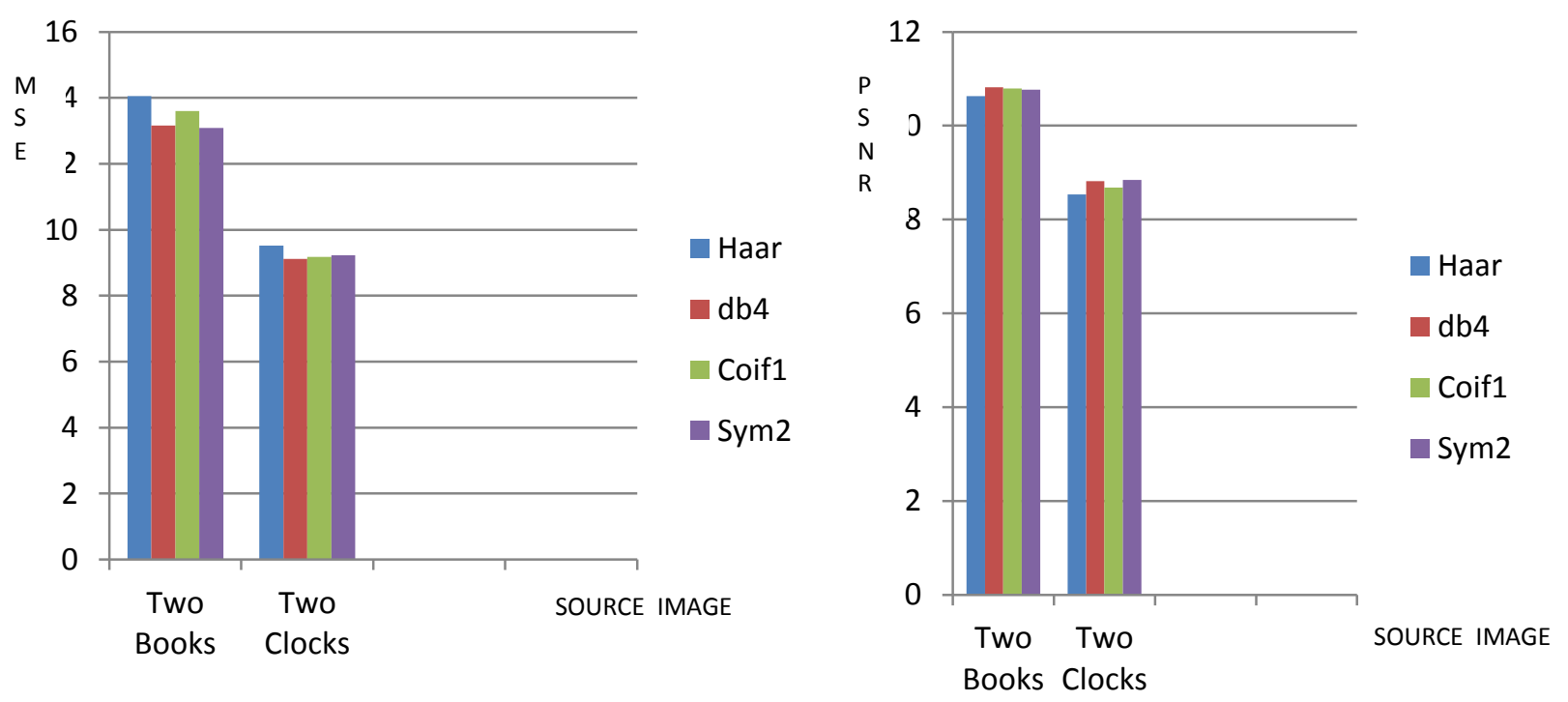

Fig No.4 shows Comparison of different image by MSE,PSNR for different types of transform. 
Table No. 1 shows the comparision of different transform by MSE,PSNR AND S.S.I.M which shows that Haar transform has maximum M.S.E and minimum P.S.N.R

\section{Conclusion}

The algorithm proposed in this paper Hybrid Image Fusion is a novel approach for fusing two images by using the different types of transform. In this two unregistered images are first registered by Geometric Transform method.In the next stage the DWT of the images is obtained ,then the fusion rules are implemented decomposed image.Then the IDWT is performed on decomposed image to obtained two fused images from which finally a hybrid image is obtained by applying fusion rules .The Hybrid fusion method. shows optimum results with haar transform as compared with other transform ,still the choice of transform depends upon the images used for fusion. Further it can be implemented by using DT-CWT method and segmentation .

\section{References}

[1] Gonzalo Pajares, Jesus Manuel De la Cruz, A Wave-based image fusion tutorial The Journal of The Pattern Recognition Society, 10.1010/j.patcog.2004.03.010.

[2] Barbara Zitova,Jan Fluseer, Image Registration Method-A Survey Image and Vision Computing 21(2003)977-1000.

[3] Medha V. Wyawahare, Dr. Pradeep M. Patil, and Hemant K. Abhyankar, Image Registration Techniques: An overview International Journal of Signal Processing, Image Processing and Pattern Recognition Vol. 2, No.3, September 2009.

[4] Jan Flusser, Filip `Sroubek, and Barbara Zitov'a, Image Fusion:Principles, Methods, and Tutorial EUSIPCO 2007 Lecture Notes

[5] Susmitha Vekkot and Pancham Shukla, A Novel Architecture for Wavelet based Image Fusion, Journal of World Academy of Science, Engineering and Technology, 2009. pp. 32-33.

[6] Stavri Nikolov, Paul Hill, David Bull, Nishan Canagarajah, WAVELETS FOR IMAGE FUSION, Image Communications GroupCentre for Communications Research University of Bristol

[7] Dr.S.S.Bedi1, Mrs.Jyoti Agarwal2, Pankaj Agarwal, Image Fusion Techniques and Quality Assessment Parameters for Clinical Diagnosis: A Review, International Journal of Advanced Research in Computer and Communication Engineering Vol. 2, Issue 2, February 2013

[8] Anjali Malviya, S.G. Bhirud, Image Fusion of Digital Images, International.Journal of Recent Trends in Engineering, Vol 2,No 3, Nov. 2009.pp.146-148. 